\title{
Théologiques
}

\section{Un regard juif sur le christianisme aujourd'hui}

\section{Armand Abécassis}

Volume 11, numéro 1-2, automne 2003

Juifs et chrétiens. L’à-venir du dialogue.

URI : https://id.erudit.org/iderudit/009535ar

DOI : https://doi.org/10.7202/009535ar

Aller au sommaire du numéro

Éditeur(s)

Faculté de théologie de l'Université de Montréal

ISSN

1188-7109 (imprimé)

1492-1413 (numérique)

Découvrir la revue

Citer cet article

Abécassis, A. (2003). Un regard juif sur le christianisme aujourd'hui.

Théologiques, 11(1-2), 261-270. https://doi.org/10.7202/009535ar d'utilisation que vous pouvez consulter en ligne.

https://apropos.erudit.org/fr/usagers/politique-dutilisation/ 


\title{
Un regard juif sur le christianisme aujourd'hui
}

\author{
Armand АвÉCASSIS \\ Département de philosophie \\ Université Michel-de-Montaigne, \\ Bordeaux III
}

\section{Pas de géant}

Il nous faut d'abord reconnaître le chemin immense parcouru par les chrétiens depuis deux millénaires, sur les plans spirituel, social et affectif. C'est grâce à eux, et au-delà de leurs maladresses, de leurs fautes et de leurs persécutions, que le monothéisme juif s'est répandu dans le monde, que toute le terre entend parler du Dieu d'Abraham, d'Isaac et de Jacob, et que les valeurs spirituelles bibliques - la justice, l'amour, la dignité humaine, la solidarité - sont devenues universelles et sont intégrées à toutes les visions du monde et à toutes les politiques. En ce sens, le christianisme participe activement à la réalisation du projet divin déposé dans la Torah et met à la disposition des hommes son éthique et sa spiritualité. Comme le judaïsme, il représente une modalité d'alliance avec Dieu, fondée sur leur commun héritage et sur le principe monothéiste.

Dès le second siècle de l'ère courante, des sectes gnostiques ont voulu dissoudre ce lien et cette continuité, en séparant la Torah des écrits saints chrétiens et purger même ceux-ci de toutes citations et de toutes références hébraïques. Mais l'Église a réagi en composant sa Bible des deux «Testaments » pour insister sur l'héritage commun des deux alliances. Depuis la Shoah, toutes les déclarations officielles faites par les chrétiens sur leur dialogue avec les Juifs, rappellent ce lien. Ainsi, le quatrième paragraphe de la Déclaration Nostra Ætate rédigée par le cardinal Béa et publiée en 1965 par le pape Paul VI ${ }^{1}$, après plusieurs

1. C'est le pape Jean XXIII qui en fut l'initiateur au Concile de Vatican II. 
révisions, commence ainsi: «Scrutant le mystère de l’Église, le concile se souvient du lien spirituel qui unit le peuple du Nouveau Testament à la lignée d'Abraham. » Ce lien spirituel est donc inscrit dans le «mystère » de l'Église, c'est-à-dire dans le dessein divin. Cela est nouveau dans l'histoire et la théologie de l'Église, qui est située ici en rapport avec le peuple juif. La Déclaration ne cesse de rappeler cette mémoire et de la signifier en s'appuyant sur Paul dont elle reprend les termes exacts: «Les Juifs restent, à cause le leurs pères, très chers à Dieu dont "les dons et l'appel sont sans repentance”.» (Rm 11,29)

Il en résulte qu'est implicitement condamnée l'idée de rejet du peuple juif par Dieu assénée par les chrétiens tout au long des siècles. La vocation d'Israël est donc reconnue comme permanente et éternelle, parallèlement à celle du christianisme, car les deux sont inscrites dans la volonté divine. Le Comité épiscopal français a abondamment explicité ce paragraphe 4 de la Déclaration ${ }^{2}$ et a demandé aux chrétiens de cesser d'interpréter l'expression «nouvel Israël » dans une perspective négative pour le peuple juif. Il la considère plus «comme un commencement que comme un aboutissement». Il aborde d'autre part, avec courage, la question du rapport d'Israël à sa terre, absente de la Déclaration et constate qu'elle met la «justice à l'épreuve». À l'occasion du $20^{\mathrm{e}}$ anniversaire de Nostra Ætate, le Saint-Siège a publié des Notes pour une correcte présentation des Juifs et du Judaïsme dans la prédication et la catéchèse de l'Église catholique $^{3}$. On peut y lire, plus fermement fondées et plus explicitement affirmées, les propositions des Déclarations antérieures: «... les juifs et le judaïsme ne devraient pas occuper une place occasionnelle et marginale dans la catéchèse et la prédication, mais leur présence indispensable doit y être intégrée de façon organique ${ }^{4}$.» Le Saint-Siège y insiste sur la ques-

2. Conférence épiscopale française, Orientations pastorales du Comité épiscopal pour les relations avec le judaïsme (16 avril 1973); texte publié dans Documentation Catholique, no 1631 (6 mai 1973) p. 419-422.

3. Commission du Saint-Siège pour les relations avec le judaĩsme, Notes pour une correcte présentation des juifs et du judaïsme dans la catéchèse et la prédication de l'Église catholique, publiées dans Documentation Catholique, $\mathrm{n}^{\circ} 1900$ (21 juillet 1985) p. 733-738.

4. «Catholiques et juifs: un nouveau regard», p. 733. À Mayence, le 17 novembre 1980, le pape JeAn-Paul II proclama devant la communauté juive: «... le peuple de Dieu de l'ancienne Alliance - une Alliance qui n'a jamais été révoquée par Dieu (cf. Rm 11, 29) » ( "Discours adressé par Jean-Paul II aux représentants de la communauté juive (Mayence, 17 novembre 1980)», Istina, 31 (1980) p. 193-194). 
tion de l'antijudaïsme chrétien, sur celle de la terre d'Israël et sur celle de la Shoah, mais il ne va malheureusement pas loin dans sa réflexion: ses insuffisances manifestent les difficultés rencontrées, traduisent le cheminement chrétien à travers les inévitables compromis, mais donnent la preuve des changements opérés dans l'Église.

À partir du pontificat de Jean-Paul II, les relations judéo-chrétiennes se sont développées dans un louable esprit d'ouverture, de reconnaissance, de respect et de fraternité. Aujourd'hui encore les images du Pape devant le mur des Lamentations font ressurgir en nous l'émotion profonde qui nous avait étreints lors de sa visite en Israël. Voir un pape à Jérusalem après deux millénaires d'existence chrétienne, ne relève pas exclusivement de l'histoire, de la politique ou de l'affectivité.

De tous ces bouleversements spirituels chez les chrétiens, nous ne pouvons, nous, Juifs, qu'être heureux, même si nos exigences et nos attentes ne nous paraissent pas entièrement satisfaites.

Mais ce n'est pas seulement sur le plan spirituel que se fondent et s'expriment les formidables avancées, parfois révolutionnaires, de la pensée et de la théologie chrétiennes. Les relations judéo-chrétiennes sur les plans affectif, social, personnel et communautaire établissent chaque jour des bases du dialogue qui ne pourront être ébranlées malgré les différences spirituelles. L'investissement des chrétiens et des Juifs dans la même aventure religieuse, dans les mêmes responsabilités bibliques et à travers les mêmes idées de création, de révélation, d'alliance universelle, de pardon et de libération, contribue à les relier les uns aux autres par l'estime, par la fraternité et même par l'admiration. Des amitiés solides et définitives se nouent tout au long des séminaires et des études en commun. La confiance du Juif face au chrétien se confirme à chacune de ces rencontres et de ces partages et ce dernier ne voit plus dans le premier un chrétien en puissance. Elle suscite le courage de développer et de multiplier ces rencontres desquelles chacun sort renforcé dans sa propre foi et enrichi par sa confrontation avec celle de l'autre. C'est pourquoi les déclarations de repentance des chrétiens, catholiques et protestants, ne peuvent pas être seulement signifiées et justifiées par des arguments intellectuels, sociaux ou théologiques. C'est le cœur du chrétien qui s'y exprime, hanté par le remords, la révolte mais par la tendresse également. Et que dire des chrétiens morts en déportation avec les Juifs, fusillés par les nazis parce qu'ils avaient aidé, logé et protégé des enfants et des 
adultes juifs. Il faudrait écrire un jour l'histoire des curés, des sœurs, des pasteurs et des simples chrétiens que nous, Juifs, reconnaissons comme des «justes et pieux» des nations. Ce sont eux qui ont témoigné de la spiritualité chrétienne authentique. Un jour arrivera où tous les chrétiens verront la continuité entre la Torah et l'enseignement de Jésus, lorsqu'on aura débarrassé celui-ci des accusations antijuives dont les apôtres l'ont chargé. Pourrait-on concevoir, lorsqu'on est un homme de la Bible, d'autres principes de la vie en commun que ceux de la justice et de l'amour du prochain... et du lointain?

\section{Des questions en souffrance}

Cependant, comme dans tout dialogue sérieux et authentique, le chrétien et le Juif ne peuvent éviter les sérieuses difficultés qu'ils rencontrent, dans leurs responsabilités respectives et dans leur solidarité au sein du projet divin dont ils se chargent quotidiennement, chacun à sa manière.

Il y a d'abord le lourd passé des souffrances et des morts infligées aux Juifs par les chrétiens, par l'Église et par les papes, parce qu'ils résistaient à toute conversion et à toute assimilation. Des préjugés de toutes sortes - politiques, psychologiques, sociaux, économiques, sexuels, et même spirituels - courent encore aujourd'hui dans l'esprit et dans le cœur des hommes comme dans les dictionnaires, à cause de très nombreux théologiens catholiques et protestants.

Il y a aussi les conduites maladroites et inopportunes du Pape qui béatifie certaines personnes dont les idées et les actions à l'égard des Juifs paraissent ambiguës ou franchement hostiles. La déclaration suivante du Pape dans son homélie de la béatification d'Edith Stein ne nous paraît pas acceptable: «... elle est morte en fille d'Israël, "glorifiant son saint Nom (le Nom du Seigneur)" et en même temps en sœur Thérèse Bénédicte de la Croix, bénie par la croix ${ }^{5}$.» Dans une même cérémonie, le Pape a béatifié Pie IX et Jean XXIII! Jean XXIII est un «juste et pieux» devant Dieu. Sa béatification honore l'Église et toute l'humanité. Pie IX a rétabli le ghetto à Rome, répété les discriminations abominables dont les Juifs étaient victimes et a trouvé légitimes les conversions forcées!

5. «Fille d'Israël bénie par la croix. Homélie pour la béatification d'Édith Stein à Cologne», Documentation Catholique, n 1941 (7 juin 1987) p. 572. 
Il y a encore la situation des lieux saints en Israël et les relations de l'État juif avec le Vatican: elle ne nous semble pas parfaitement clarifiée.

Il y a bien sûr le problème des "Palestiniens» sur lequel de très nombreux chrétiens se prononcent de manière unilatérale et donc injuste.

Il y a ensuite l'attitude des chrétiens devant les informations tronquées, et même truquées, des médias concernant le événements politiques du Moyen-Orient d'une part, et les communautés juives d'autre part. Nous attendons des chrétiens qu'ils réagissent fermement contre l'antijudaïsme qui se développe chaque jour dans le monde.

\section{Questions théologiques}

Il y a enfin et surtout les questions religieuses et théologiques. "Il y a beaucoup à faire dans la liturgie, dans la lecture de la Bible et dans les commentaires qui en sont donnés, en éducation, en catéchèse, dans la prédication, pour une meilleure connaissance de l'autre et un meilleur respect de ce qu'il est en vérité ${ }^{6}$.» Telle est l'avant-dernière phrase du livre du père Jean Dujardin, L'Église catholique et le peuple juif. Un autre regard.

En effet, frères chrétiens, qu'attendez-vous de nous, Juifs ? Vous avez enfin compris que Dieu ne vous demandait pas de nous convertir à votre religion issue pourtant du judaïsme. Il a élu Israël et lui a donné la Torah, en lui demandant de rester fidèle à sa parole qui y est inscrite. Nous avons notre manière de la lire, de l'interpréter, de la pratiquer, d'y croire et de la transmettre. Elle est écrite dans notre langue et elle contient notre mémoire, depuis la création jusqu'au temps du scribe Ezra. La loi orale, qui caractérise la spiritualité pharisienne au sein de laquelle Jésus fut formé, instruit et éduqué, nous enseigne les modalités d'appropriation du texte révélé (le Pentateuque), inspiré (les Prophètes) ou rédigé par des sages (les Écrits). Nous vous demandons de découvrir notre modalité d'appropriation du texte biblique et d'apprendre à lire vos textes saints à la lumière de la Torah, alors qu'on vous a appris pendant deux millénaires à lire la Torah à la lumière de vos Écrits saints. On vous a convaincus que le christianisme était «l'accomplissement des Écritures et la

6. J. Dujardin, L'Église catholique et le peuple juif. Un autre regard (Diaspora), Paris, Calmann-Lévy, 2003, p. 388. 
fleur du judaïsme» selon la métaphore botanique. De cette modalité d'appropriation que vous avez faite de notre Torah sont issues des conceptions ambiguës et très précipitamment enfermées dans des expressions telles que "nouvel Israël ", «nouveau peuple de Dieu », "Nouveau Testament» et donc "Ancien Testament», qui reposent sur le schéma de la substitution. Les chrétiens conscients et responsables les déplorent et les corrigent, mais combien sont-ils encore à les comprendre sans dévalorisation ni réprobation du judaïsme et des Juifs? Le peuple juif n'a pas été choisi par Dieu pour le préparer à produire Jésus et le christianisme même si, historiquement, celui-ci lui a succédé. Ces conceptions et ces expressions, transformées en formules décisives à la racine de la foi chrétienne, doivent être réexaminées par les Juifs et par les chrétiens ensemble, dans des séances d'études communes, dans le face à face des confrontations et des contradictions, avec respect et estime réciproques. Votre nouveau regard sur nous, Juifs, est à ce prix, de même que votre conscience de l'exacte mesure de votre tâche et de votre mission dans le monde.

Comment pouvez-vous comprendre l'originalité du message de Jésus si vous ignorez au sein de quelle culture et au milieu de quelles crises graves du judaïsme il a vécu ? Il parlait à ses frères juifs exclusivement. Le Fils de Dieu aurait pu témoigner de l'universalité de son message en s'incarnant dans le corps de n'importe quel païen. Pourquoi a-t-il choisi d'emprunter le corps d'un Juif circoncis, fréquentant le Temple de Jérusalem et les synagogues, y enseignant la Torah de Moïse et l'interprétant à la manière pharisienne ou essénienne ? L'expérience montre que le chrétien qui étudie sérieusement la Torah et se familiarise avec les pratiques juives, apporte à sa foi plus de lumière, plus de richesse et plus de conviction. Il en résulte que le judaïsme ne peut être pour vous simplement l'une des religions qui existent dans le monde: il est inscrit dans votre âme de manière privilégiée, fondatrice de votre spiritualité. Votre amour pour le peuple juif est spécifique et il est à distinguer de votre amour pour les autres cultures et religions. C'est pourquoi votre critique lucide et objective à son égard ne peut être qu'amicale et fraternelle, dans l'esprit des critiques des prophètes eux-mêmes.

Nous voudrions avoir plus de clarté sur les énoncés suivants tirés des déclarations sur les Juifs faites par les chrétiens: 
On s'efforcera de mieux comprendre ce qui, dans l'Ancien Testament, garde une valeur propre et perpétuelle (cf. Dei Verbum, 14-15), celle-ci n'étant pas oblitérée par l'interprétation ultérieure du Nouveau Testament qui lui donne sa signification plénière ${ }^{7} . .$.

Jésus [...] en se révélant comme Messie et Fils de Dieu (cf. Mt 16, 16), porteur d'un nouveau message, celui de l'Évangile, s'est présenté comme accomplissant et parachevant la Révélation antérieure ${ }^{8}$.

Dieu, inspirateur et auteur des livres des deux Testaments, s'y est pris si sagement que le Nouveau Testament était caché dans l'Ancien, et que l'Ancien devenait clair dans le Nouveau'.

«En vertu de sa mission divine, l'Église », qui est «moyen général de salut » et en laquelle seule se trouve "toute la plénitude des moyens de salut " (Unit. Red. 3), "par nature doit annoncer Jésus Christ au monde» (Orientations et Suggestions, 1). En effet nous croyons que c'est par Lui que nous allons au Père (cf. Jn 1, 6) et que «la vie éternelle c'est qu'ils te connaissent toi, le seul véritable Dieu, et ton envoyé Jésus Christ» (Jn 17, 3) ${ }^{10}$.

On leur apprendrait [aux chrétiens] ainsi à les [les Juifs] apprécier et à les aimer, eux qui ont été choisis par Dieu pour préparer la venue du Christ $^{11} \ldots$

Le Christ est désormais référence-clé des Écritures ${ }^{12} \ldots$

Avant de juger ces énoncés, il nous faut les comprendre: dans quel esprit leurs auteurs les ont-ils écrits? Nous ne pouvons accepter, fondés sur l'amour pour les Juifs et pour le judaïsme, qu'ils impliquent que la religion chrétienne est l'accomplissement de la Torah. Le christianisme a eu pour vocation fondamentale d'ouvrir les païens aux valeurs bibliques,

7. Commission du SAint-Siège Pour les Relations AVec le Judaïsme, Orientations et suggestions pour l'application de la déclaration conciliaire "Nostra Aetate », no 4, paru dans Documentation Catholique, no 1668 (19 janvier 1975) p. $59-62$ (ici, S II: «liturgie», p. 60).

8. Commission du SAINT-SiÈge, Orientations et suggestions, $\mathbb{S}$ III : « Enseignement et éducation", p. 60.

9. Constitution dogmatique Dei Verbum, $\mathbb{S} 16$, citée dans Commission du SAInTSIĖGE, Orientations et suggestions, $\mathbb{S}$ III : «Enseignement et éducation », p. 60.

10. Commission du Saint-Siège, Notes pour une correcte présentation, I, 7.

11. Commission du SAInt-Siège, Notes pour une correcte présentation, I, 8.

12. Commission du SAINT-SiÈge, Notes pour une correcte présentation, II, 5. 
certes, mais les Juifs qui possédaient la Torah depuis 12 siècles n'avaient nul besoin d'être convertis. Hors de l'Église il y a encore le salut pour les Juifs et pour les autres. Le chrétien, en tant que tel, a raison de croire que «nul ne va au Père si ce n'est par le Fils» (voir Jn 14,6). Mais les Juifs vont au Père par la Torah, par la Loi écrite lue à travers la Loi orale, en un mot: par l'interprétation du Livre (midrash).

\section{Deux chocs incontournables}

Deux grandes difficultés rendent laborieux et ardu le dialogue entre les Juifs et les chrétiens: le retour des Juifs sur leur terre et la Shoah. Nous attendons encore des chrétiens qu'ils nous disent la place qu'ils réservent dans leur théologie à ces deux événements décisifs $\mathrm{du} \mathrm{xx}^{\mathrm{e}}$ siècle pour l'histoire d'Israël et pour l'humanité. Quelle est donc la mission du peuple juif dans le plan divin d'après les chrétiens? Et quel sens donnent-ils, par ailleurs, à la souffrance juive? Les réponses à ces deux questions montrent que les chrétiens ne peuvent faire l'économie du judaïsme tel que les Juifs en témoignent par la parole et par les rites quotidiens.

Inversement, il est légitime de chercher à savoir quelle est la mission des chrétiens dans le plan divin d'après la Synagogue. Pouvons-nous, nous, Juifs, faire l'économie de la vocation chrétienne dans la compréhension de nous-mêmes et dans la mission dont le Créateur nous a chargés depuis le Sinaï?

Nous avouons humblement que, par rapport aux avancées extraordinaires de l'Église pendant les cinquante années qui ont suivi la Shoah, les communautés juives restent en retard - nous pensons aux déclarations ecclésiales et aux engagements de nombreux chrétiens, prêtres ou laïcs, que nous connaissons et qui nous ont spirituellement et affectivement enrichis. Les chrétiens nous interpellent, nous, Juifs. Ne laissons pas s'écrouler par notre faute le magnifique édifice de compréhension et d'amour construit depuis la Shoah. Comme l'affirme la Déclaration Dabru Emet du 10 septembre 2000 ${ }^{13}$, nous devons œuvrer ensemble pour la justice et pour la paix. Nos racines sont antérieures à notre division en deux alliances différentes, les deux nécessaires pour faire des-

13. Déclaration juive sur les chrétiens et le christianisme rédigée par des universitaires et des rabbins américains. Voir DujARdin, L'Église catholique et le peuple juif, p. 480-483 et, dans le présent numéro, les articles de L. KLEnicki et de M.A. Signer. 
cendre la sainteté dans le monde. Si, après l'échec de toutes les idéologies en Occident, les Juifs et les chrétiens ne se donnent pas la main pour en témoigner, qui pourrait s'en charger? Ne voit-on pas que le dialogue véritable entre eux, doit servir de paradigme à tous les autres dialogues des chrétiens et des Juifs avec les autres religions, avec les religieux et avec les non-religieux sur la planète.

Heureusement, certains Juifs qui connaissent leur histoire et qui s'appuient sur des maîtres prestigieux, considèrent le christianisme comme un véritable monothéisme malgré le dogme trinitaire. À l'exemple des Tossafot, de Rabbenou Menahem Ha-Méiri, de Judah Hallévi, de Bahia Ibn Paqouda, de Maïmonide, de Nahmanide, de Ralbag, du Maharal de Prague, ils croient que le projet divin se réalise aussi grâce aux chrétiens qui ont largement contribué à améliorer l'humanité, à lui transmettre une éthique authentique et à faire connaître les paroles de la Torah par toutes les nations. Judah Hallévi a écrit que le christianisme «... ne fait que frayer la voie pour le Roi-Messie et pour améliorer le monde entier afin qu'il serve Dieu d'un commun accord ${ }^{14} »$ (Kuzari 4,23).

Les Juifs et les chrétiens ont pour mission de convertir l'homme, non pas au christianisme ou au judaïsme, mais à lui-même d'abord et à Dieu ensuite. Il doivent lui apprendre à aller à Dieu par ses propres voies. Leur tâche est difficile et deviendrait impossible s'ils ignoraient eux-mêmes ce que signifie aller à Dieu au-delà de leurs voies respectives. Jésus a-t-il enseigné à ses apôtres de convertir les hommes au christianisme ou de s'appuyer sur son témoignage pour les orienter vers le Dieu de tous?

Pour la tradition juive, «les justes et les pieux des nations ont part au monde à venir ».

14. Judah Hallévi, Le Kuzari, apologie de la religion mépriséel trad. par C. Touati, avec intro. et notes, Louvain/Paris, Peeters, 1994. 


\section{RÉSUMÉ}

Comment fonder et préparer un véritable dialogue entre Juifs et chrétiens devant le même Père? La première condition en est la connaissance réciproque: le chrétien doit se dépouiller de tous les préjugés répandus contre le judaïsme et se mettre à l'étudier et à l'interroger sérieusement. Le Juif également doit apprendre à découvrir le christianisme authentique en se débarrassant de ses préjugés et de sa méfiance à son égard. L'autre condition est la conviction des Juifs et des chrétiens que Dieu a besoin de ces deux alliances pour réaliser son projet pour le monde et pour l'humanité: deux alliances irréductibles l'une à l'autre et pourtant complémentaires et nécessaires dans leurs relations dialectiques de paix et d'amour.

\section{ABSTRACT}

Sharing the same God, Jews and Christians are willing to established a true dialog. How can this be really achieved? A first condition is that they get a better knowledge of one another: Christians have to get rid of all current prejudice against Judaism and must start to study it and to ask serious questions about it; Jews, setting aside any bias and mistrust, must also learn to discover the authentic face of Christianity. Second, Jews and Christians should share the conviction that God needs each of their covenants to bring his plan on the world and on mankind to completion. None of these covenants can be reduced to the other; on the contrary, they supplement each other and are both necessary in their dialectic relations of peace and love.

(C) Revue Théologiques 2003. Tout droit réservé. 